\title{
DNA vaccines for cervical cancer: from bench to bedside
}

\author{
Chien-Fu Hung ${ }^{1,2}$, Archana Monie ${ }^{1}$, \\ Ronald D. Alvarez ${ }^{5}$ and T.-C. $\mathrm{Wu}^{1,2,3,4,6}$ \\ ${ }^{1}$ Department of Pathology \\ ${ }^{2}$ Department of Oncology \\ ${ }^{3}$ Department of Obstetrics and Gynecology \\ ${ }^{4}$ Department of Molecular Microbiology and Immunology \\ Johns Hopkins Medical Institutions \\ Baltimore, MD 21231, USA \\ ${ }^{5}$ Department of Gynecology and Obstetrics \\ University of Alabama at Birmingham \\ Birmingham, AL 35249, USA \\ ${ }^{6}$ Corresponding author: Tel, 1-410-614-3899; \\ Fax, 1-443-287-4295; E-mail, wutc@jhmi.edu
}

Accepted 18 October 2007

Abbreviations: CIN, cervical intraepithelial neoplasia; DC, dendritic cell; EGCG, epigallocatechin-3-gallate; EGFP, enhanced GFP; ER, endoplasmic reticulum; GFP, green fluorescent protein; HPV, human papillomavirus; LAMP-1, lysosomal-associated membrane protein type 1; PADRE, pan HLA-DR binding epitope; VLP, virus-like particle; XIAP, x-linked inhibitor of apoptosis protein

\begin{abstract}
More than $99 \%$ of cervical cancers have been associated with human papillomaviruses (HPVs), particularly HPV type 16. The clear association between HPV infection and cervical cancer indicates that HPV serves as an ideal target for development of preventive and therapeutic vaccines. Although the recently licensed preventive HPV vaccine, Gardasil, has been shown to be safe and capable of generating significant protection against specific HPV types, it does not have therapeutic effect against established HPV infections and HPV-associated lesions. Two HPV oncogenic proteins, E6 and E7, are consistently co-expressed in HPV-expressing cervical cancers and are important in the induction and maintenance of cellular transformation. Therefore, immunotherapy targeting E6 and/or E7 proteins may provide an opportunity to prevent and treat HPV-associated cervical malignancies. It has been established that $T$ cell-mediated immunity is one of the most crucial components to defend against HPV infections and HPV-associated lesions. Therefore, effective therapeutic HPV vaccines should generate strong E6/E7-specific $T$ cell-mediated immune responses. DNA vaccines have emerged as an attractive
\end{abstract}

approach for antigen-specific T cell-mediated immunotherapy to combat cancers. Intradermal administration of DNA vaccines via a gene gun represents an efficient way to deliver DNA vaccines into professional antigen-presenting cells in vivo. Professional antigen-presenting cells, such as dendritic cells, are the most effective cells for priming antigen-specific $T$ cells. Using the gene gun delivery system, we tested several DNA vaccines that employ intracellular targeting strategies for enhancing MHC class I and class II presentation of encoded model antigen HPV-16 E7. Furthermore, we have developed a strategy to prolong the life of DCs to enhance DNA vaccine potency. More recently, we have developed a strategy to generate antigen-specific $\mathrm{CD}^{+} \mathrm{T}$ cell immune responses to further enhance DNA vaccine potency. The impressive preclinical data generated from our studies have led to several HPV DNA vaccine clinical trials.

Keywords: antigen presenting cells; dendritic cells; human papillomavirus type 16; oncogene protein E7; papillomavirus vaccines; vaccination; vaccines, DNA

\section{Introduction}

Cervical cancer is an important disease because it is the $2^{\text {nd }}$ largest cause of cancer deaths in women worldwide (Parkin et al., 2005). It has been established that the primary factor in the development of cervical cancer is persistent infection with human papillomavirus (HPV) and HPV DNA has been detected in $99.7 \%$ of cervical cancers (Walboomers et al., 1999). More than 200 HPV genotypes have been identified and they are classified into low or high-risk types, depending on their propensity to cause cervical cancer (de Villiers et al., 2004). High-risk types, such as HPV 16 and HPV 18, are the most frequent HPV type associated with cervical cancer. High-risk HPV types are frequently associated with squamous intraepithelial lesions (SIL), also called cervical intraepithelial neoplasia (CIN) precursor lesions of cervical cancer (For a review, see Roden and Wu, 2006).

The molecular biology of human papillomavirus has been well studied. HPV has a circular, doublestranded DNA genome containing about 8,000 base pairs and encoding two classes of proteins: early proteins and late proteins. The early proteins regulate viral DNA replication (E1, E2), viral RNA transcription (E2), cytoskeleton reorganization (E4) 
and cell transformation (E5, E6, E7), whereas the late proteins $(\mathrm{L} 1, \mathrm{~L} 2)$ are structural components of the viral capsid. The expression of these proteins is tightly regulated and associated with the differentiation of infected epithelial cells. E2 functions as the master regulator that regulates the expression of all the other viral genes, and is particularly involved in the repression of E6 and $E 7$. The viral oncogenes, E6 and E7 are responsible for transformation. In most cases of cervical cancer, the HPV genome integrates into the host chromosomal DNA and leads to the disruption of the viral E2 gene. Since E2 is a transcriptional repressor of E6 and E7, loss of E2 leads to upregulation of E6 and E7 genes. The uncontrolled expression of E6 and E7 proteins disrupts normal cell cycle regulation by interacting with $\mathrm{p} 53$ and $\mathrm{Rb}$ thereby prolonging the cell cycle and suppressing apoptosis, contributing to the progression of HPV-associated cervical cancer (For a review, see zur Hausen, 2002).

A basic understanding of HPV biology is essential for the development of vaccines against HPV. Vaccination represents a highly cost-effective approach to reduce the mortality of HPV. The clear association between HPV infection and cervical cancer indicates that HPV serves as an ideal target for development of preventive and therapeutic vaccines. Vaccination could be implemented to prevent infection by generating neutralizing antibodies to block HPV viral infection (preventive vaccines) or to eliminate infection by inducing a virus-specific $T$ cell-mediated response (therapeutic vaccines). The structural components of infectious viral capsids, L1 and L2 proteins have been extensively used as targets for preventive vaccine development. These vaccines act by generating neutralizing antibodies against HPV infection. However, L1 and L2 are not expressed in the basal cells infected with HPV, unlike the HPV early proteins, such as E6 and E7 which are expressed early in viral infection at all levels of the infected epithelium and help regulate the progression of the disease. Therefore, therapeutic vaccines should aim to generate $T$ cell-mediated immune responses against the early proteins, such as E6 and E7.

The Food and Drug Administration (FDA) recently approved a novel preventive HPV vaccine, Gardasil, developed by Merck for the control of HPV infections. This vaccine uses HPV virus-like particles (VLP) to generate neutralizing antibodies against major capsid protein, L1. However, prophylactic HPV vaccines do not have therapeutic effects against pre-existing HPV infections and HPV-associated lesions. Furthermore, due to the considerable burden of HPV infections worldwide, it would take decades for preventive vaccines to impact the prevalence of cervical cancer. Thus, in order to further facilitate the control of cervical cancer and to address patients with current infections, the continued development of therapeutic vaccines against HPV is critical. Therapeutic HPV vaccines can potentially eliminate preexisting lesions and malignant tumors by generating cellular immunity against HPV-infected cells.

The major factor involved in the designing of therapeutic vaccines is the choice of target antigen. In case of HPV, the early viral proteins such as E6 and E7 are potentially ideal target antigens since they are expressed early in viral infection. Furthermore, the genes E6 and E7 are essential for transformation and are co-expressed in HPV infected cells but not in normal cells (Crook et al., 1989). Thus, these genes represent suitable target antigens for the development of therapeutic HPV vaccines.

There are a number of therapeutic vaccine approaches mainly targeting E6 and E7, that have been tested in preclinical and clinical trials, such as peptide or protein-based vaccines, live vector vaccines, cell-based vaccines and DNA vaccines. Among different forms of therapeutic HPV vaccines, DNA vaccines have become an attractive and potentially effective strategy for antigen- specific immunotherapy. Naked DNA is relatively safe, stable, and easy to produce, and can be used to sustain high levels of antigen expression in cells (for reviews, see Donnelly et al., 1997; Gurunathan et al., 2000). In addition, since DNA vaccines do not elicit antibodies against DNA in the patient, they can be repeatedly administered. DNA vaccines, however, are poorly immunogenic because DNA lacks cell type specificity. Furthermore, DNA lacks the intrinsic ability to amplify or spread to surrounding cells in vivo. The potency of DNA vaccines can be enhanced by targeting DNA or encoded antigen to professional APCs and by modifying the properties of antigen-expressing APCs in order to boost vaccine-elicited immune responses.

Professional APCs, especially dendritic cells (DCs), are the central players in the initiation of the adaptive immune response. Accumulating evidence suggests that cell-mediated immunity is critical for the control of viral infections and malignant tumors. It is now clear that DCs play a key role in the generation of antigen-specific antiviral and antitumor $T$ cell immune responses. The generation of $\mathrm{CD}^{+} \mathrm{T}$ cells allows for direct killing of viral-infected cells or tumors, while the generation of $\mathrm{CD}^{+} \mathrm{T}$ helper cells leads to the augmentation of both $\mathrm{CD}^{+}$immune responses. DCs are chiefly responsible for the presentation of antigen 
to naïve $\mathrm{CD}^{+}$and $\mathrm{CD} 8^{+} \mathrm{T}$ cells and for the activation of armed effector $\mathrm{CD} 4^{+}$and $\mathrm{CD} 8^{+} \mathrm{T}$ cells. Immature DCs, which are located in peripheral tissues, efficiently uptake antigens, process the antigens into antigenic peptides, and load the peptides onto MHC class I and class II molecules in order to present them on the cell surface. These immature DCs possess various types of surface receptors which enable them to respond to danger signals, such as bacterial or viral components or inflammatory cytokines indicating the presence of an infection (for a review, see Guermonprez et al., 2002). In response to a danger signal, the DCs undergo a maturation process, upregulating adhesion and co-stimulatory molecules and transforming into efficient APCs and potent activators of $T$ cells. These DCs then migrate to the lymphoid organs to select and stimulate antigen-specific $T$ cells (for reviews, see Steinman, 1991; Banchereau et al., 2000; Guermonprez et al., 2002).

Therefore, we have developed various strategies to enhance the potency of DNA vaccines and to augment vaccine-elicited $T$ cell immune responses; these strategies have focused on: 1) increasing the number of antigen-expressing DCs; 2) improving antigen expression, processing, and presentation in DCs and; 3) enhancing DC and T cell interaction. The current review discusses the various strategies to enhance DNA vaccine potency and their eventual clinical translation.

\section{Strategies to increase the number of antigen-expressing and/or antigen-loaded DCs}

\section{Intradermal administration of DNA vaccines via gene gun as an efficient route for the delivery of DNA to DCs}

One strategy for increasing antigen-expressing DC populations is to find convenient and effective routes for the delivery of DNA vaccines directly into DCs in vivo. To date, DNA vaccines have been most commonly administered via either the intramuscular or intradermal route (for a review, see Gurunathan et al., 2000). Among the previously explored routes of DNA administration, intradermal vaccination via a gene gun represents one of the most efficient methods for delivering DNA directly into DCs. The gene gun can deliver DNA-coated gold particles into intradermal Langerhans cells in vivo (Condon et al., 1996); these cells then mature and migrate to the lymphoid organs for $\mathrm{T}$ cell priming (Porgador et al., 1998). In addition, gene gun immunization is significantly more dose-efficient than intramuscular or subcutaneous injection (for reviews, see Gurunathan et al., 2000; Payne et al., 2002). Thus, intradermal administration of DNA by gene gun allows us to test several strategies that require the delivery of DNA directly into dendritic cells. We have used this system to modify the properties of dendritic cells to enhance DNA vaccine potency (for a review, see Hung and Wu, 2003).

\section{Intercellular antigen spreading as a strategy to increase the number of antigen-expressing DCs}

The potency of DNA vaccines is still limited by the inability of naked DNA to naturally amplify and spread among DCs in vivo. This limitation can potentially be overcome by promoting the spread of encoded antigen from transfected cells to DCs. Linkage of antigen to proteins capable of intercellular transport has been shown to facilitate the spread of encoded antigen. For example, we have previously employed the herpes simplex virus type 1 (HSV-1) tegument protein VP22 for our DNA vaccine development. When HSV-1 VP22 is linked to thymidine kinase (Dilber et al., 1999), cytosine deaminase (Wybranietz et al., 2001), p53 (Phelan et al., 1998), or green fluorescent protein (GFP) (Elliott and O'Hare, 1997), it has been shown to distribute these proteins to the nuclei of surrounding cells. A fusion gene consisting of VP22 linked to a model antigen, human papillomavirus type 16 (HPV-16) E7, was demonstrated to dramatically increase the number of E7-expressing DCs in the lymph nodes of mice (Kim et al., 2004b) as well as enhance E7-specific long-term memory $\mathrm{CD} 8^{+} \mathrm{T}$ cell immune responses and antitumor effects against E7-expressing tumor cells (Hung et al., 2001a; Kim et al., 2004b). Similarly, HSV-1 VP22 has been employed in a DNA vaccine to enhance vaccineelicited immune responses against HPV-16 E6 (Peng et al., 2005) as well as influenza NP antigen (Saha et al., 2006).

Two other proteins with some homology to VP22, bovine herpesvirus VP22 (BVP22) and Marek's disease virus VP22 (MVP22) have also been shown to be capable of intercellular spreading and transport (Koptidesova et al., 1995; Dorange et al., 2000; Harms et al., 2000; Mwangi et al., 2005). We have shown that vaccination with a fusion gene consisting of MVP22 linked to HPV-16 E7 enhances E7-specific $\mathrm{CD}^{+} \mathrm{T}$ cell responses and antitumor effects in mice relative to immunization with wild-type E7 DNA (Hung et al., 2002).

Some concerns are raised that the intercellular trafficking ability of VP22 may potentially be attributed to fixation artifacts (Lundberg and Johansson, 2001). For example, one group found that transfection of mammalian cells with DNA 
encoding HSV-1 VP22 fused to enhanced GFP (EGFP) did not lead to increased intercellular spread of the fusion protein in vitro, but could lead to significantly enhanced production of EGFPspecific antibodies in mice vaccinated with this construct (Perkins et al., 2005). However, the transport properties of VP22 without fixation have been demonstrated (Oliveira et al., 2001). In particular, binding and transport of an RNA $\left(\cup_{s} 8.5-\right.$ EGFP) into surrounding cells by VP22 without fixation has been shown (Sciortino et al., 2002). Since no intercellular transport of $U_{s} 8.5$-EGFP mRNA was observed in the absence of VP22, this study supports the conclusion that VP22 can move between cells. To date, the ability of VP22 to bind and transport non- $U_{s} 8.5$-EGFP mRNA has not been shown. However, if these issues are addressed, it is possible that in the future co-administration of DNA encoding VP22 and DNA encoding antigen of interest may represent a method for the distribution of antigen to surrounding cells.

\section{Linkage of antigen to molecules capable of binding to DCs as a method to target antigen to DCs}

Another strategy to increase the number of antigen-expressing or antigen-loaded DCs is the linkage of antigen to molecules that target the antigen to the surface of DCs in the context of DNA vaccines. For example, DNA vaccines encoding antigen linked to a secreted form of heat shock protein 70 , which bind to scavenger receptors on the surface of DCs such as CD91, may represent an effective method for targeting linked antigen to DCs and enhancing antigen-specific immunity (Hauser and Chen, 2003; Trimble et al., 2003; Hauser et al., 2004). We have also shown that linkage of antigen to Fms-like tyrosine kinase 3-ligand can also target antigen to DCs; a chimeric FIt-3L-E7 fusion gene can significantly improve CTL responses in mice compared to vaccination with wild-type E7 DNA (Hung et al., 2001c).

\section{Employment of chemotherapy-induced apoptotic cell death to increase the number of antigen-loaded DCs}

Co-administration of DNA vaccines with chemotherapeutic agents in the presence of an established tumor can promote the release of antigen from apoptotic tumor cells. This can potentially facilitate antigen uptake by local DCs, resulting in enhancement of DNA vaccine potency. For example, we have recently shown that the chemotherapeutic agent epigallocatechin-3-Gallate (EGCG), a chemical derived from green tea, could induce tumor cellular apoptosis and enhance the tumor antigen-specific $T$ cell immune responses elicited by DNA vaccination (Kang et al., 2007). However, it is important to note that such a strategy is more appropriate in a therapeutic context (i.e. in the presence of a tumor) because in the absence of a tumor, EGCG actually leads to decreased DNA vaccine potency, presumably acting as an suppressor of DC function (Kang et al., 2007). It may be of interest to explore whether other chemotherapeutic agents could exhibit similar synergistic effects when combined with DNA vaccines.

\section{Strategies to improve antigen expression, processing, and presentation in DCs}

\section{Codon optimization as a strategy to enhance antigen expression in DCs}

Codon optimization has emerged as an important strategy to enhance the expression of encoded antigen in DCs. This technique involves the modification of antigenic gene sequences by replacement of codons that are rarely recognized by cellular protein synthesis machinery with more commonly recognized codons. This strategy can enhance the translation of DNA vaccines in DCs. For example, we have demonstrated that immunization of mice with codon-optimized HPV-16 E6 DNA (Lin et al., 2006) generated greater immune responses than vaccination of mice with wild-type E6 DNA.

\section{Employment of intracellular targeting strategies to enhance MHC class I and class II antigen presentation in DCs}

Increased progress in our understanding of intracellular pathways for antigen presentation has created the opportunity to enhance DNA vaccine potency. Strategies to facilitate MHC class I antigen processing in DCs can lead to the activation of larger populations of $\mathrm{CD}^{+} \mathrm{T}$ cells and the generation of stronger antitumor or antiviral immunity. It has been demonstrated that linkage of antigen to proteins that target the antigen for proteasomal degradation or entry into the endoplasmic reticulum (ER) can facilitate MHC class I presentation of linked antigen in DCs. For example, linkage of antigen to Mycobacterium tuberculosis heat shock protein 70 (hsp70) (Chen et al., 2000a), $\gamma$-tubulin (Hung et al., 2003), calreticulin (CRT) (Cheng et al., 2001) or the translocation domain of Pseudomonas aeruginosa exotoxin A [ETA(dII)] (Hung et al., 2001b) can significantly improve MHC class I 


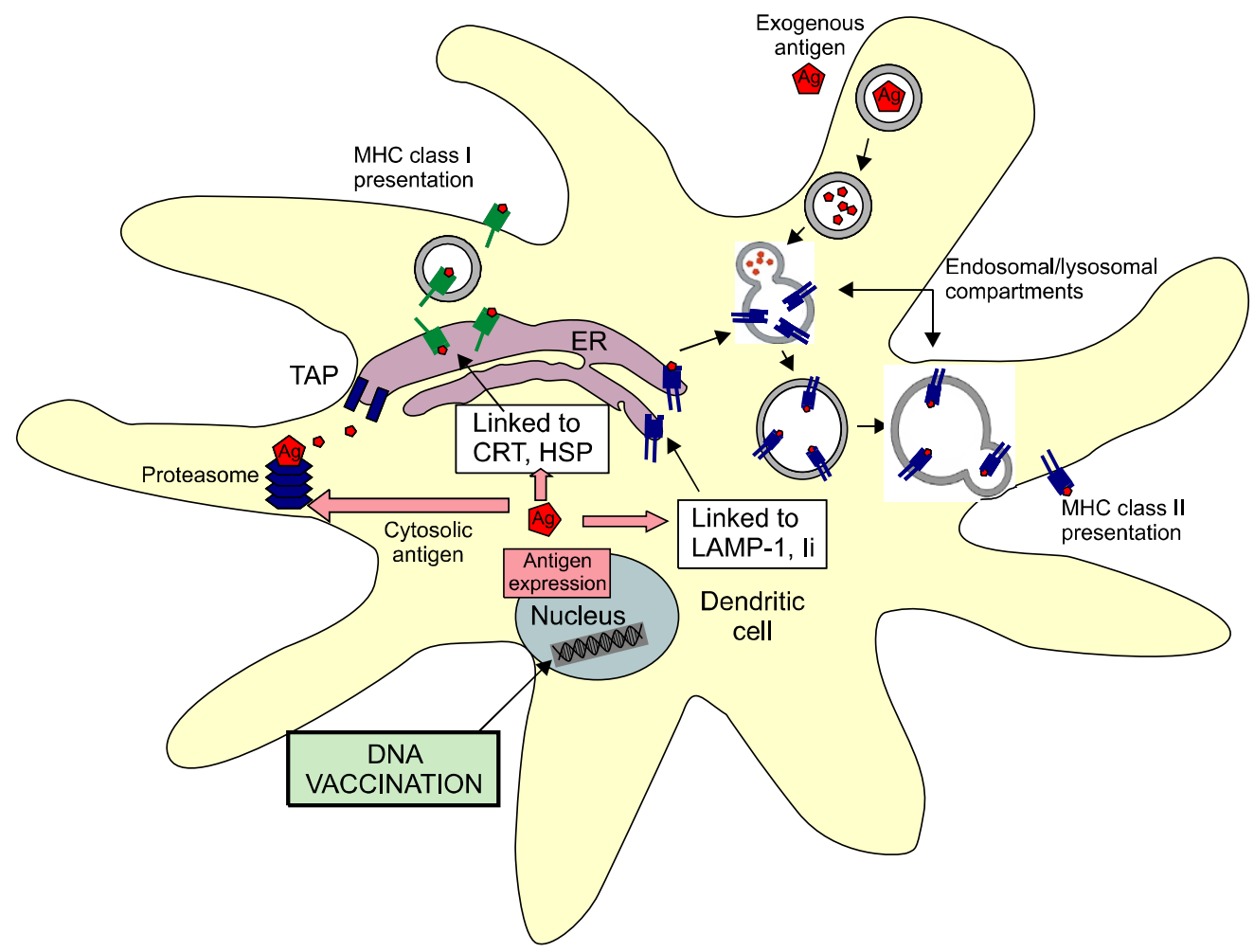

Figure 1. Antigen presentation pathways in DCs. In the MHC class I presentation pathway, cytosolic antigens are targeted for proteasomal degradation. The resulting antigenic peptides associate with MHC class I molecules in the ER and are transported to the cell surface. In the MHC class II pathway, exogenous antigens are endocytosed and the vesicle containing antigen is fused with lysosomes, facilitating antigen degradation. The resulting vesicle is then fused with a vesicle containing MHC class II molecules, and the $\mathrm{MHC}$ class II molecule/antigenic peptide complexes are transported to the surface of the cell. In the context of DNA vaccines, linkage of antigen to HSP70 or CRT targets antigen for entry into the ER, enhancing MHC class I presentation of the antigen. Linkage of antigen to LAMP-1 of li targets the antigen to the MHC class II processing pathway. The peptide-MHC complex is then routed to the endosomal/lysosomal compartments for improved class II antigen presentation. DC, dendritic cell; MHC, major histocompatibility complex; ER, endoplasmic reticulum; HSP70, heat shock protein 70; CRT, calreticulin; LAMP-1, lysosomal-associated membrane protein type 1; li, MHC class II-associated invariant chain.

presentation of nuclear or cytosolic antigens (see Figure 1). In a head-to-head comparison of HPV16 E7 DNA vaccines employing intracellular targeting strategies, we found that a DNA vaccine encoding E7 linked to CRT generated the greatest E7-specific CTL responses and antitumor effects against $E 7$-expressing tumors in a preclinical model (Kim et al., 2004a). The encouraging results from these preclinical studies have led to several proposed HPV DNA vaccine clinical trials at Johns Hopkins (Dr. T.-C. Wu, personal communications).

Strategies have also been developed to enhance MHC class II presentation of antigen encoded by DNA vaccines. It is increasingly evident that $\mathrm{CD4}^{+}$helper $\mathrm{T}$ cells also play a major role in the priming of $\mathrm{CD}^{+} \mathrm{T}$ cells as well as the generation of memory $T$ cells (Castellino and Germain, 2006). Thus, strategies to facilitate MHC class II presentation of vaccine-encoded antigen can significantly improve the potency of the DNA vaccines. For example, we have demonstrated that fusion of
HPV-16 E7 with the sorting signal of lysosomalassociated membrane protein type 1 (LAMP-1) can target the E7 antigen to cellular endosomal/lysosomal compartments and facilitate class II presentation of E7 (Wu et al., 1995) (See Figure 1). This construct was tested in the context of a DNA vaccine and generated higher numbers of E7specific $\mathrm{CD}^{+}{ }^{\mathrm{T}}$ cells as well as greater E7-specific CTL activity in mice than DNA vaccines encoding wild-type $\mathrm{E} 7$ alone (Ji et al., 1999).

More recently, we have developed a DNA vaccine strategy employing MHC class II-associated invariant chain (li) for improving class II presentation of antigen in DCs. In the ER, li binds with MHC class II molecules, and the class IIassociated peptide (CLIP) region of li occupies the peptide-binding groove of the MHC molecule, preventing premature binding of antigenic peptides into the groove (for reviews, see Cresswell, 1994; Trombetta and Mellman, 2005). In the endosomal/ lysosomal compartments, CLIP is replaced by a 


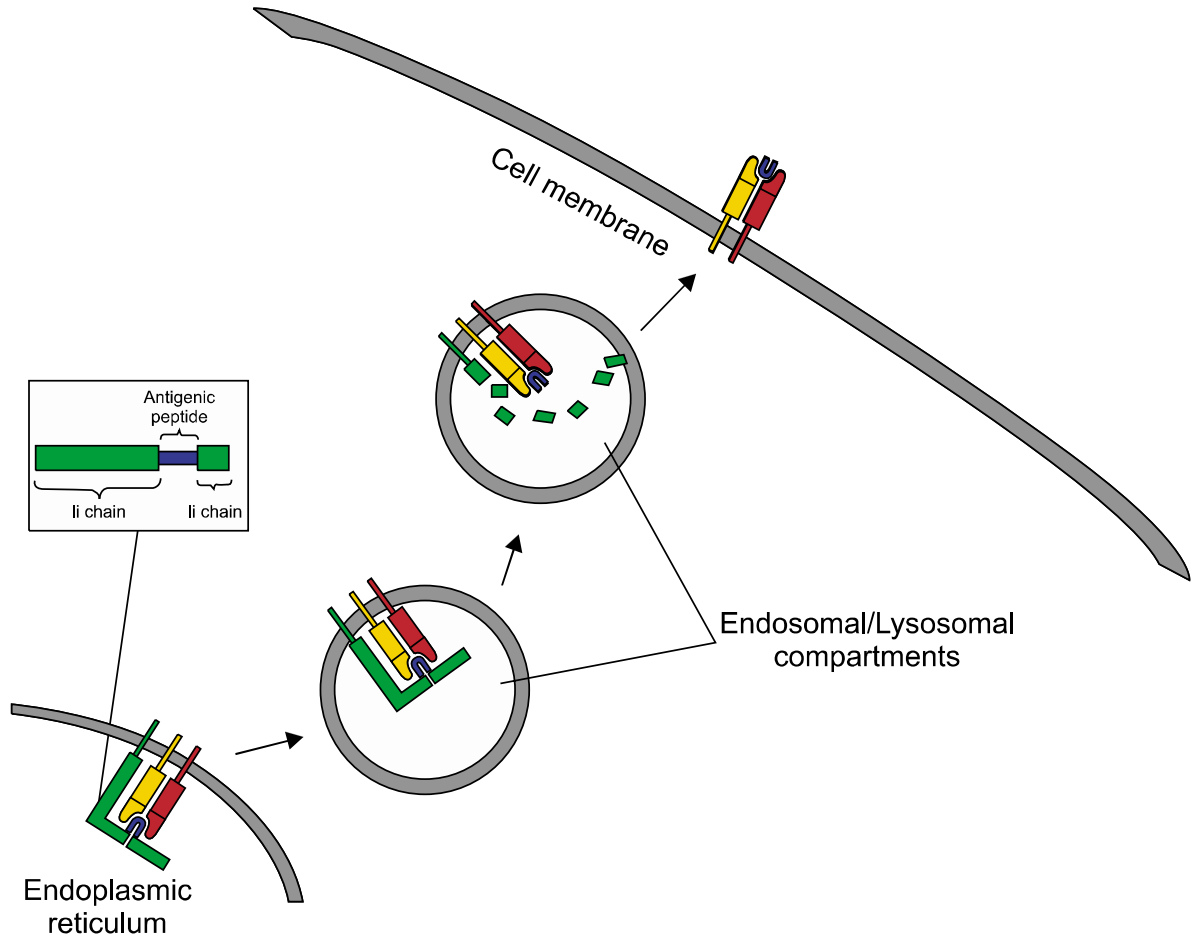

Figure 2. Employment of li for stable presentation of a desired antigenic peptide by DCs. The translated li-antigen peptide enters the ER and binds to an MHC class II molecule, with the antigenic peptide occupying the peptidebinding groove of the MHC molecule. In the lysosomal compartments, the li chain is degraded, leaving the desired antigenic peptide in the peptide-binding groove of the MHC class II molecule and allowing for the stable presentation of the antigenic peptide on the cell surface. peptide antigen, and the MHC class II molecule/ antigenic peptide complex is then presented on the cell surface. By substituting the CLIP region of Ii with a desired epitope, the epitope can be efficiently presented through the MHC class II pathway in DCs for the stimulation of peptidespecific $\mathrm{CD}^{+} \mathrm{T}$ cells (see Figure 2). Using this rationale, we have created a DNA vaccine encoding li with the CLIP region replaced with the pan HLA-DR binding epitope (PADRE) and shown that this DNA vaccine could elicit potent PADREspecific $C D 4^{+} \mathrm{T}$ cell responses in vaccinated mice (Hung et al., 2007b). In addition, a co-administration of this vaccine (termed li-PADRE) with DNA encoding HPV-16 E7 generated significantly greater $\mathrm{CD}^{+}{ }^{+} \mathrm{T}$ cell immune responses relative to a co-administration of DNA encoding HPV-16 E7 with DNA encoding unmodified li (Hung et al., 2007b).

\section{Bypassing antigen processing as a method for generating stable antigen presentation in DCs}

We have recently employed a MHC class I singlechain trimer (SCT) technology to bypass the antigen processing and presentation. This strategy involves the linkage of the gene encoding an E6 antigenic peptide to $\beta 2$-microglobulin and a $\mathrm{MHC}$ class I heavy chain, producing a single-chain construct encoding the peptide antigen fused to an MHC class I molecule (see Figure 3). The expres-
A



B

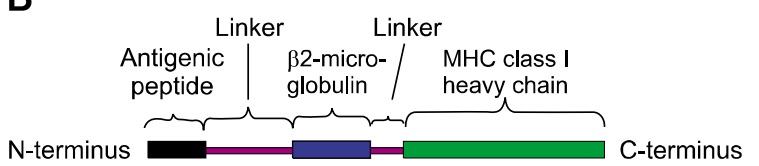

Figure 3. (A) Illustration of a SCT (comprising antigenic peptide, $\beta 2$-microglobulin, and MHC class I heavy chain) on the cell surface. (B) The SCT gene construct.

sion of this construct within DCs may allow for stable presentation of the E6 antigenic peptide through MHC class I molecules on the cell surface. We have shown that a DNA vaccine encoding a SCT encoding a HPV-16 E6 CTL epitope, $\beta 2-$ 
microglobulin, and $\mathrm{H}-2 \mathrm{~K}^{\mathrm{b}} \mathrm{MHC}$ class I heavy chain generated enhanced E6 peptide-specific $\mathrm{CD}^{+} \mathrm{T}$ cell responses in vaccinated mice relative to immunization with DNA encoding wild-type HPV-16 E6 (Huang et al., 2005). In addition, such strategies have also been applied to enhance DNA vaccine potency in other antigenic systems (Hung et al., 2007a).

\section{Strategies to enhance $\mathrm{DC}$ and $\mathrm{T}$ cell interaction}

\section{Prolonging $\mathrm{DC}$ survival to enhance $\mathrm{T}$ cell interaction}

After T cell priming, DCs may become targets of $T$ cell-mediated apoptosis. Thus, strategies to prolong the life of DCs may further increase the number of activated T cells. We have developed a strategy to prolong DC survival using DNA encoding antiapoptotic proteins, including $\mathrm{Bcl}-\mathrm{xL}$, $\mathrm{Bcl}-2$, X-linked inhibitor of apoptosis protein (XIAP), and dominant negative mutants $(\mathrm{dn})$ of caspases such as $d n$ caspase- 9 and $d n$ caspase- 8 . Our studies have indicated that co-administration of a DNA vaccine encoding HPV-16 E7 with DNA encoding any of these antiapoptotic factors could augment E7-specific CTL responses and elicit potent antitumor effects against E7-expressing tumors in vaccinated mice (Kim et al., 2003). However, delivery of DNA encoding antiapoptotic proteins into cells raises concerns for cellular malignant transformation. The use of gene knockdown strategies such as RNA interference (RNAi) targeting key proapoptotic proteins may provide a novel method for accomplish the same goal while alleviating the concern of oncogenicity. Thus, we have shown that co-administration of a DNA vaccine encoding HPV-16 E7 with siRNA targeting the key proapoptotic proteins Bak and Bax can prolong the survival of transfected DCs and augment E7-specific $\mathrm{CD}^{+} \mathrm{T}$ cell responses against E7-expressing tumors in mice (Kim et al., 2005). These studies suggest that prolongation of DC survival may represent an effective strategy for improving antigen presentation by $\mathrm{DCs}$ to $\mathrm{T}$ cells and for enhancing vaccine-elicited $T$ cell immune responses. It would be of interest to further explore co-administration of DNA vaccines with siRNA targeting other key proapoptotic proteins, such as caspase-8, caspase-9 and/or caspase-3, to enhance DNA vaccine potency.

\section{Induction of $\mathrm{CD}^{+} \mathrm{T}$ cell help as a strategy for augmenting $\mathrm{CD8}^{+} \mathrm{T}$ cell responses}

The activation of $\mathrm{CD}^{+} \mathrm{T}$ cells is dependent upon, and augmented by, $\mathrm{T}$ cell help from $\mathrm{CD}^{+} \mathrm{T}$ cells. Thus, strategies to induce $\mathrm{CD}^{+} \mathrm{T}$ helper cells at sites of $\mathrm{CD}^{+} \mathrm{T}$ cell priming can potentially enhance CTL immune responses. For example, we have shown that co-administration of DNA vaccines with DNA encoding an li chain with the CLIP region replaced with pan HLA-DR binding epitope (PADRE) (see above) could lead to significant enhancement in antigen-specific $\mathrm{CD}^{+}$ immune responses (Hung et al., 2007b). This suggests a potentially effective vaccine strategy for $\mathrm{CD}^{+} \mathrm{T}$ cell-mediated augmentation of $\mathrm{CD} 8^{+} \mathrm{T}$ cell priming for potentiating DNA vaccines. Furthermore, the strategy to enhance $\mathrm{CD}^{+} \mathrm{T}$ cell help can be used in conjunction with other strategies, such as strategies to prolong the life of DCs and/or other intracellular targeting strategies to further enhance DNA vaccine potency, since these strategies are not mutually exclusive (Kim et al., 2007).

\section{DNA vaccine clinical trials}

The encouraging data from preclinical models have led to several therapeutic HPV DNA vaccine clinical trials. For example, DNA vaccines encoding a signal sequence linked to an attenuated form of HPV-16 E7 (with a mutation that abolishes the Rb binding site) (E7detox) and fused to heat shock protein 70 (Sig/E7detox/Hsp70) are currently undergoing phase I trials on HPV-16 positive patients with high-grade CIN lesions at Johns Hopkins (Dr. Cornelia Trimble, personal communication). The trial design was a straight dose-escalation phase I, testing a homologous DNA-DNA-DNA prime-boost vaccination regimen of three vaccinations per patient, at three dose levels, 500, 1,000, and 3,000 micrograms, delivered intramuscularly at one-month intervals, prior to standard therapeutic resection of remaining lesion, seven weeks after the last vaccination. No adverse or dose-limiting site effects were observed at any dose level of the DNA vaccine and the vaccination was considered to be feasible and tolerable in patients with CIN2/3 lesions. The patients in the highest dose cohort generated IFN- $\gamma$ secreting $\mathrm{CD}^{+} \mathrm{T}$ cell immune responses to $\mathrm{E} 7$ of greater magnitude in PBMCs than subjects in lower dose cohorts. Thus, it appears to be possible to generate antigen-specific cytotoxic $T$ cells with this construct, in patients with established CIN2/3 lesions. Disease regression was not observed at the first two dose levels in the 15-week study window. In the highest dose cohort, however, disease regression was observed in 3 of 9 patients post-vaccination. The analysis regarding the correlation of the immune response with lesion regression is 
currently ongoing. Another phase I trial using the same naked DNA vaccine (Sig/E7detox/ Hsp70) is currently ongoing in HPV-16 positive patients with advanced head and neck squamous cell carcinoma (Dr. Maura Gillison, personal communication) at the Johns Hopkins University. Likewise, no significant adverse effects were observed in this study. Some of the DNA treated patients developed appreciable E7-specific immune responses.

Another candidate DNA vaccine that is currently being prepared for clinical trials conducted at University of Alabama at Birmingham in collaboration with Johns Hopkins is a DNA vaccine encoding calreticulin (CRT) fused to HPV-16 E7 (E7detox) (Drs. Ronald Alvarez and Cornelia Trimble, personal communication). Intradermal administration of the CRT/E7 DNA vaccine has been shown to generate significant E7 antigen-specific immune responses in preclinical models (see above). This therapeutic HPV DNA vaccine trial will be performed in HPV-16 positive patients with stage 1B1 cervical cancer using a PowderMed/ Pfizer proprietary gene gun device ND-10, which is an individualized gene gun device suitable for clinical trials. This study aims to investigate whether the repeated, cluster (short-interval) intradermal CRT/ E7 DNA vaccination is safe and able to generate E7-specific $\mathrm{CD}^{+} \mathrm{T}$ cell immune responses in patients with stage 1B1 resectable cervical cancer. DNA vaccination using the cluster vaccination regimen has been shown to rapidly induce antigenspecific $\mathrm{CD}^{+} \mathrm{T}$ cell immune responses in preclinical models (Dr. T.-C. Wu, personal communication). The proposed trial using cluster vaccination regimen will make it possible to complete the vaccination regimen before tumor resection allowing the assessment of the influence of the DNA vaccination on the tumor microenvironment without compromising the standard care of the patient.

\section{Combination vaccines}

The effect of therapeutic HPV DNA vaccines may be enhanced through a combination approach using heterologous prime-boost strategies. Prime- boost regimens are perhaps the most effective treatment strategy for vaccination against HPV. Because nucleic acid vaccines often generate relatively weak CTL responses, combinatorial vaccination approaches are used to circumvent this limitation. Priming with a DNA or RNA vaccine and then boosting with a viral vector vaccine has been shown to result in enhanced immune responses relative to single modality vaccinations. For example, we have demonstrated that vaccination with DNA prime fol- lowed by vaccinia boost regimen generates a significantly higher antigen-specific immune response compared to DNA vaccination alone (Chen et al., 2000b). A phase II clinical trial using heterologous E7 DNA prime (Sig/E7(detox)/HSP70) followed by vaccinia boost (TA-HPV) is currently being planned in HPV-16 associated CIN2/3 patients at Johns Hopkins (Dr. Cornelia Trimble, personal communication). TA-HPV is a vaccinia construct derived from the Wyeth strain of vaccinia, which has been obtained from Xenova/Cantab/ Celtic Pharma and has been shown to be less neurovirulent than the parental virus. TA-HPV was engineered to express the E6 and E7 genes from HPV types 16 and 18 (Boursnell et al., 1996). The proposed phase II clinical trial will also include the topical administration of Toll-like receptor agonist, imiquimod to enhance access of the effector immune cells to the intraepithelial compartments of lesions. Toll-like receptor agonists, such as imiquimod have been shown to activate the immature DCs and contribute to the direct killing of tumor cells (Stary et al., 2007). Thus, our clinical trial design will test whether the combination of pNGVL4a-Sig/E7 (detox)/HSP70 DNA prime-TA-HPV vaccinia boost vaccination with or without imiqimod treatment is safe and well-tolerated in patients with HPV-16 associated $\mathrm{CIN} 2 / 3$ lesions. Furthermore, the trial will determine if the combination of DNA prime-TA-HPV vaccinia boost vaccination with imiqimod treatment will generate significantly stronger E7-specific immune response and better therapeutic effects compared to prime-boost vaccination alone or imiquimod treatment alone.

Combination approaches including chemotherapy, radiation or other biotherapeutic agents combined with HPV therapeutic vaccination may also serve to enhance the therapeutic HPV vaccine potency. For example, a recent study has shown that the chemotherapeutic agent epigallocatechin3-Gallate (EGCG), a chemical derived from green tea, could induce tumor cellular apoptosis and enhance the tumor antigen-specific T cell immune responses elicited by DNA vaccination (Kang et al., 2007). These successful results have led to the planning of a phase I clinical trial at Johns Hopkins involving the combination of oral EGCG administration with intradermal administration of CRT/E7 DNA vaccination via gene gun in patients with advanced HPV-associated head and neck squamous cell carcinomas (HPV-HNSCC) (Dr. Sara Pai, personal communication).

The therapeutic effects of HPV vaccines may be further enhanced by combination with blocking the factors that inhibit T cell activation, such as CTLA4 and PD-1. These molecules are negative core- 
gulators in the T-cell costimulatory pathway. Thus, antibody-mediated blockade of CTLA-4 and PD-1 can potentially be used to prolong antitumoral $T$ cell responses (for reviews, see Peggs et al., 2006; Blank and Mackensen, 2007). The combination of HPV therapeutic vaccines with agents that influence the tumor microenvironment may also potentially be used to generate enhanced therapeutic effects against HPV-associated malignancies. It is now clear that several factors present in the tumor microenvironment may potentially hinder immunotherapy. These factors include the expression of B7-H1 (Goldberg et al., 2007), STAT3 (Yu et al., 2007) and MIC-A and B (Groh et al., 2002), indoleamine 2,3-dioxygenase (IDO) enzyme (Munn and Mellor, 2004), and galectin-1 (Rubinstein et al., 2004) on tumor cells, immunosuppressive cytokines such as IL-10 (Yue et al., 1997) and TGF- $\beta$ (Gorelik and Flavell, 2001), T regulatory cells (Curiel et al., 2004), myeloid-derived suppressor cells (Nagaraj et al., 2007). It is conceivable that agents capable of blocking these molecules may potentially be used to enhance the therapeutic effects of the HPV vaccines.

\section{Conclusions}

The identification and characterization of high-risk human papillomavirus as a necessary causal agent for cervical cancer provides a promising possibility for the eradication of HPV-related malignancies. In the development of therapeutic HPV DNA vaccines, we have focused on enhancing DNA vaccine potency and to augment vaccine-elicited $T$ cell immune responses by: 1) increasing the number of antigen-expressing DCs via gene gun; 2) improving antigen expression, processing, and presentation in DCs; and 3) enhancing DC and T cell interaction. These strategies can potentially be combined to further enhance DNA vaccine potency. Furthermore, it is important for HPV therapeutic DNA vaccines to consider using strategies such as prime-boost regimens and/or combinations strategies using molecules that are capable of blocking the negative regulators on $\mathrm{T}$ cells to further enhance the $\mathrm{T}$ cell immune responses. Moreover, increasing understanding of the molecular mechanisms that hinder immune attack in the tumor microenvironment will lead to the identification of novel molecular targets that can be blocked in order to enhance the therapeutic effect of HPV DNA vaccines. With continued endeavor in the development of HPV therapeutic vaccines, we can foresee that HPV therapeutic DNA vaccines will emerge as a significant approach that can be combined with existing forms of therapy such as chemotherapy and radiation leading to effective translation from bench to bedside for the control of HPV-associated malignancies.

\section{Acknowledgement}

This review is not intended to be an encyclopedic one, and the authors apologize to those not cited. We would like to thank Drs. Richard Roden and Tae-Woo Kim for their critical review of the manuscript. The work is supported by the NCI SPORE in Cervical Cancer P50 CA098252 and $\mathrm{NCl}$ 1RO1 CA114425-01.

\section{References}

Banchereau J, Briere F, Caux C, Davoust J, Lebecque S, Liu YJ, Pulendran B, Palucka K. Immunobiology of dendritic cells. Annual review of immunology 2000;18:767-811

Blank C, Mackensen A. Contribution of the PD-L1/PD-1 pathway to T-cell exhaustion: an update on implications for chronic infections and tumor evasion. Cancer Immunol Immunother 2007;56:739-45

Boursnell ME, Rutherford E, Hickling JK, Rollinson EA, Munro AJ, Rolley N, McLean CS, Borysiewicz LK, Vousden $\mathrm{K}$, Inglis SC. Construction and characterisation of a recombinant vaccinia virus expressing human papillomavirus proteins for immunotherapy of cervical cancer. Vaccine 1996;14:1485-94

Castellino F, Germain RN. Cooperation between CD4 ${ }^{+}$and $\mathrm{CD}^{+} \mathrm{T}$ cells: when, where, and how. Annual review of immunology 2006;24:519-40

Chen C-H, Wang T-L, Hung C-F, Yang Y, Young RA, Pardoll DM, Wu T-C. Enhancement of DNA vaccine potency by linkage of antigen gene to an HSP70 gene. Cancer Research 2000a;60:1035-42

Chen CH, Wang TL, Hung CF, Pardoll DM, Wu TC. Boosting with recombinant vaccinia increases HPV-16 E7-specific T cell precursor frequencies of HPV-16 E7-expressing DNA vaccines. Vaccine 2000b;18:2015-22

Cheng WF, Hung CF, Chai CY, Hsu KF, He L, Ling M, Wu TC. Tumor-specific immunity and antiangiogenesis generated by a DNA vaccine encoding calreticulin linked to a tumor antigen. J Clin Invest 2001;108:669-78

Condon C, Watkins SC, Celluzzi CM, Thompson K, Falo LD Jr. DNA-based immunization by in vivo transfection of dendritic cells. Nat Med 1996;2:1122-8

Cresswell P. Assembly, transport, and function of MHC class II molecules. Annual review of immunology 1994;12:259-93

Crook T, Morgenstern JP, Crawford L, Banks L. Continued expression of HPV-16 E7 protein is required for maintenance of the transformed phenotype of cells co-transformed by HPV-16 plus EJ-ras. Embo J 1989;8:513-9 
Curiel TJ, Coukos G, Zou L, Alvarez X, Cheng P, Mottram P, Evdemon-Hogan M, Conejo-Garcia JR, Zhang L, Burow M, Zhu Y, Wei S, Kryczek I, Daniel B, Gordon A, Myers L, Lackner A, Disis ML, Knutson KL, Chen L, Zou W. Specific recruitment of regulatory $T$ cells in ovarian carcinoma fosters immune privilege and predicts reduced survival. Nat Med 2004;10: 942-9

de Villiers EM, Fauquet C, Broker TR, Bernard HU, zur Hausen H. Classification of papillomaviruses. Virology 2004; 324:17-27

Dilber MS, Phelan A, Aints A, Mohamed AJ, Elliott G, Smith $\mathrm{Cl}$, O'Hare $\mathrm{P}$. Intercellular delivery of thymidine kinase prodrug activating enzyme by the herpes simplex virus protein, VP22. Gene Ther 1999;6:12-21

Donnelly JJ, Ulmer JB, Shiver JW, Liu MA. DNA vaccines. Annual review of immunology 1997;15:617-48

Dorange F, El Mehdaoui S, Pichon C, Coursaget P, Vautherot JF. Marek's disease virus (MDV) homologues of herpes simplex virus type 1 UL49 (VP22) and UL48 (VP16) genes: high-level expression and characterization of MDV-1 VP22 and VP16. J Gen Virol 2000;81 Pt 9:2219-30

Elliott G, O'Hare P. Intercellular trafficking and protein delivery by a herpesvirus structural protein. Cell 1997;88: 223-33

Goldberg MV, Maris CH, Hipkiss EL, Flies AS, Zhen L, Tuder RM, Grosso JF, Harris TJ, Getnet D, Whartenby KA, Brockstedt DG, Dubensky TW Jr, Chen L, Pardoll DM, Drake CG. Role of PD-1 and its ligand, $\mathrm{B} 7-\mathrm{H} 1$, in early fate decisions of CD8 T cells. Blood 2007;110:186-92

Gorelik L, Flavell RA. Immune-mediated eradication of tumors through the blockade of transforming growth factor-beta signaling in T cells. Nat Med 2001;7:1118-22

Groh V, Wu J, Yee C, Spies T. Tumour-derived soluble MIC ligands impair expression of NKG2D and T-cell activation. Nature 2002;419:734-8

Guermonprez P, Valladeau J, Zitvogel L, Thery C, Amigorena $\mathrm{S}$. Antigen presentation and $\mathrm{T}$ cell stimulation by dendritic cells. Annual Review of Immunology 2002;20:621-67

Gurunathan S, Klinman DM, Seder RA. DNA vaccines: immunology, application, and optimization. Annual Review of Immunology 2000;18:927-74

Harms JS, Ren X, Oliveira SC, Splitter GA. Distinctions between bovine herpesvirus 1 and herpes simplex virus type 1 VP22 tegument protein subcellular associations. J Virol 2000;74:3301-12

Hauser $\mathrm{H}$, Chen SY. Augmentation of DNA vaccine potency through secretory heat shock protein-mediated antigen targeting. Methods 2003;31:225-31

Hauser H, Shen L, Gu QL, Krueger S, Chen SY. Secretory heat-shock protein as a dendritic cell-targeting molecule: a new strategy to enhance the potency of genetic vaccines. Gene Ther 2004;11:924-32

Huang CH, Peng S, He L, Tsai YC, Boyd DA, Hansen TH, Wu TC, Hung CF. Cancer immunotherapy using a DNA vaccine encoding a single-chain trimer of MHC class I linked to an HPV-16 E6 immunodominant CTL epitope. Gene Ther 2005;

\section{$12: 1180-6$}

Hung C-F, Cheng W-F, Chai C-Y, Hsu K-F, He L, Ling M, Wu T-C. Improving vaccine potency through intercellular spreading and enhanced MHC class I presentation of antigen. J Immunol 2001a;166:5733-40

Hung C-F, Cheng W-F, Hsu K-F, Chai C-Y, He L, Ling M, Wu T-C. Cancer immunotherapy using a DNA vaccine encoding the translocation domain of a bacterial toxin linked to a tumor antigen. Cancer Research 2001b;61:3698-703

Hung C-F, Hsu K-F, Cheng W-F, Chai CY, He L, Ling M, Wu T-C. Enhancement of DNA vaccine potency by linkage of antigen gene to a gene encoding the extracellular domain of Flt3-ligand. Cancer Research 2001c;61:1080-8

Hung CF, He L, Juang J, Lin TJ, Ling M, Wu TC. Improving DNA vaccine potency by linking Marek's disease virus type 1 VP22 to an antigen. J Virol 2002;76:2676-82

Hung CF, Cheng WF, He L, Ling M, Juang J, Lin CT, Wu TC. Enhancing major histocompatibility complex class I antigen presentation by targeting antigen to centrosomes. Cancer Res 2003;63:2393-8

Hung CF, Wu TC. Improving DNA vaccine potency via modification of professional antigen presenting cells. Curr Opin Mol Ther 2003;5:20-4

Hung C-F, Calizo R, Tsai YC, He L, Wu T-C. A DNA vaccine encoding a single-chain trimer of HLA-A2 linked to human mesothelin peptide generates anti-tumor effects against human mesothelin-expressing tumors. Vaccine 2007a;25: 127-35

Hung CF, Tsai YC, He L, Wu TC. DNA vaccines encoding li-PADRE generates potent PADRE-specific CD4(+) T-cell immune responses and enhances vaccine potency. Mol Ther 2007b;15:1211-9

Ji H, Wang T-L, Chen C-H, Hung C-F, Pai S, Lin K-Y, Kurman RJ, Pardoll DM, Wu T-C. Targeting HPV-16 E7 to the endosomal/lysosomal compartment enhances the antitumor immunity of DNA vaccines against murine HPV-16 E7expressing tumors. Hum Gene Ther 1999;10:2727-40

Kang TH, Lee JH, Song CK, Han HD, Shin BC, Pai SI, Hung CF, Trimble C, Lim JS, Kim TW, Wu TC. Epigallocatechin-3gallate enhances CD8+ T cell-mediated antitumor immunity induced by DNA vaccination. Cancer Res 2007;67:802-11

Kim D, Hoory T, Wu T-C, Hung CF. Enhancing DNA Vaccine Potency by Combining a Strategy to Prolong Dendritic Cell Life and Intracellular Targeting Strategies with a Strategy to Boost CD4+ T cells. Human Gene Therapy 2007;18:1129-40

Kim JW, Hung CF, Juang J, He L, Kim TW, Armstrong DK, Pai SI, Chen PJ, Lin CT, Boyd DA, Wu TC. Comparison of HPV DNA vaccines employing intracellular targeting strategies. Gene Ther 2004a;11:1011-8

Kim TW, Hung CF, Ling M, Juang J, He L, Hardwick JM, Kumar S, Wu T-C. Enhancing DNA vaccine potency by co-administration of DNA encoding anti-apoptotic proteins. $\mathrm{J}$ Clin Invest 2003;112:109-17

Kim TW, Hung CF, Kim JW, Juang J, Chen PJ, He L, Boyd DA, Wu TC. Vaccination with a DNA vaccine encoding herpes simplex virus type 1 VP22 linked to antigen generates 
long-term antigen-specific CD8-positive memory T cells and protective immunity. Hum Gene Ther 2004b;15:167-77

Kim TW, Lee JH, He L, Boyd DA, Hardwick JM, Hung CF, Wu TC. Modification of professional antigen-presenting cells with small interfering RNA in vivo to enhance cancer vaccine potency. Cancer Res 2005;65:309-16

Koptidesova D, Kopacek J, Zelnik V, Ross NL, Pastorekova $\mathrm{S}$, Pastorek J. Identification and characterization of a cDNA clone derived from the Marek's disease tumour cell line RPL1 encoding a homologue of alpha-transinducing factor (VP16) of HSV-1. Arch Virol 1995;140:355-62

Lin CT, Tsai YC, He L, Calizo R, Chou HH, Chang TC, Soong YK, Hung CF, Lai $\mathrm{CH}$. A DNA vaccine encoding a codonoptimized human papillomavirus type $16 \mathrm{E} 6$ gene enhances CTL response and anti-tumor activity. J Biomed Sci 2006;13:481-8

Lundberg M, Johansson M. Is VP22 nuclear homing an artifact? Nat Biotechnol 2001;19:713-4

Munn DH, Mellor AL. IDO and tolerance to tumors. Trends Mol Med 2004;10:15-8

Mwangi W, Brown WC, Splitter GA, Zhuang Y, Kegerreis K, Palmer GH. Enhancement of antigen acquisition by dendritic cells and MHC class II-restricted epitope presentation to CD4+ T cells using VP22 DNA vaccine vectors that promote intercellular spreading following initial transfection. J Leukoc Biol 2005;78:401-11

Nagaraj S, Gupta K, Pisarev V, Kinarsky L, Sherman S, Kang L, Herber DL, Schneck J, Gabrilovich DI. Altered recognition of antigen is a mechanism of CD8+ T cell tolerance in cancer. Nat Med 2007;13:828-35

Oliveira SC, Harms JS, Afonso RR, Splitter GA. A genetic immunization adjuvant system based on BVP22-antigen fusion. Hum Gene Ther 2001;12:1353-9

Parkin DM, Bray F, Ferlay J, Pisani P. Global cancer statistics, 2002. CA Cancer J Clin 2005;55:74-108

Payne LG, Fuller DH, Haynes JR. Particle-mediated DNA vaccination of mice, monkeys and men: looking beyond the dogma. Curr Opin Mol Ther 2002;4:459-66

Peggs KS, Quezada SA, Korman AJ, Allison JP. Principles and use of anti-CTLA4 antibody in human cancer immunotherapy. Curr Opin Immunol 2006;18:206-13

Peng S, Trimble C, Ji H, He L, Tsai YC, Macaes B, Hung CF, Wu TC. Characterization of HPV-16 E6 DNA vaccines employing intracellular targeting and intercellular spreading strategies. J Biomed Sci 2005;12:689-700

Perkins SD, Hartley MG, Lukaszewski RA, Phillpotts RJ, Stevenson FK, Bennett AM. VP22 enhances antibody responses from DNA vaccines but not by intercellular spread. Vaccine 2005;23:1931-40

Phelan A, Elliott G, O'Hare P. Intercellular delivery of functional p53 by the herpesvirus protein VP22. Nat Biotechnol 1998; $16: 440-3$

Porgador A, Irvine KR, Iwasaki A, Barber BH, Restifo NP, Germain RN. Predominant role for directly transfected dendritic cells in antigen presentation to CD8+ T cells after gene gun immunization. J Exp Med 1998;188:1075-82

Roden R, Wu TC. How will HPV vaccines affect cervical cancer? Nat Rev Cancer 2006;6:753-63

Rubinstein N, Alvarez M, Zwirner NW, Toscano MA, Ilarregui JM, Bravo A, Mordoh J, Fainboim L, Podhajcer OL, Rabinovich GA. Targeted inhibition of galectin-1 gene expression in tumor cells results in heightened T cell- mediated rejection; a potential mechanism of tumor-immune privilege. Cancer Cell 2004;5:241-51

Saha S, Yoshida S, Ohba K, Matsui K, Matsuda T, Takeshita F, Umeda K, Tamura Y, Okuda K, Klinman D, Xin KQ, Okuda $\mathrm{K}$. A fused gene of nucleoprotein (NP) and herpes simplex virus genes (VP22) induces highly protective immunity against different subtypes of influenza virus. Virology 2006; 354:48-57

Sciortino MT, Taddeo B, Poon AP, Mastino A, Roizman B. Of the three tegument proteins that package mRNA in herpes simplex virions, one (VP22) transports the mRNA to uninfected cells for expression prior to viral infection. Proc Natl Acad Sci USA 2002;99:8318-23

Stary G, Bangert C, Tauber M, Strohal R, Kopp T, Stingl G. Tumoricidal activity of TLR7/8-activated inflammatory dendritic cells. J Exp Med 2007;204:1441-51

Steinman RM. The dendritic cell system and its role in immunogenicity. Annual review of immunology 1991;9:271-96

Trimble C, Lin CT, Hung CF, Pai S, Juang J, He L, Gillison M, Pardoll D, Wu L, Wu TC. Comparison of the CD8+ T cell responses and antitumor effects generated by DNA vaccine administered through gene gun, biojector, and syringe. Vaccine 2003;21:4036-42

Trombetta ES, Mellman I. Cell biology of antigen processing in vitro and in vivo. Annual review of immunology 2005;23: 975-1028

Walboomers JM, Jacobs MV, Manos MM, Bosch FX, Kummer JA, Shah KV, Snijders PJ, Peto J, Meijer CJ, Munoz $\mathrm{N}$. Human papillomavirus is a necessary cause of invasive cervical cancer worldwide. J Pathol 1999;189:12-9

Wu T-C, Guarnieri FG, Staveley-O'Carroll KF, Viscidi RP, Levitsky HI, Hedrick L, Cho KR, August T, Pardoll DM. Engineering an intracellular pathway for $\mathrm{MHC}$ class II presentation of HPV-16 E7. Proc Natl Acad Sci 1995;92: 11671-5

Wybranietz WA, Gross CD, Phelan A, O'Hare P, Spiegel M, Graepler F, Bitzer M, Stahler P, Gregor M, Lauer UM. Enhanced suicide gene effect by adenoviral transduction of a VP22-cytosine deaminase (CD) fusion gene. Gene Ther $2001 ; 8: 1654-64$

Yu H, Kortylewski M, Pardoll D. Crosstalk between cancer and immune cells: role of STAT3 in the tumour microenvironment. Nat Rev Immunol 2007;7:41-51

Yue FY, Dummer R, Geertsen R, Hofbauer G, Laine E, Manolio S, Burg G. Interleukin-10 is a growth factor for human melanoma cells and down-regulates HLA class-I, HLA class-II and ICAM-1 molecules. Int J Cancer 1997;71:630-7

zur Hausen $\mathrm{H}$. Papillomaviruses and cancer: from basic studies to clinical application. Nat Rev Cancer 2002;2:342-50 